\title{
ANALISIS DE LA DISTRIBUCION ESPACIAL DE LOS GLACIARES ROCOSOS EN EL PIRINEO CENTRAL OSCENSE
}

\author{
Javier CHUECA CIA
} Universidad de Zaragoza

\begin{abstract}
Resumen.- Un total de 170 glaciares rocosos fueron identificados en un inventario realizado a partir del análisis de fotografias aéreas de un área superior a los $6.500 \mathrm{~km}^{2}$ que incluye la parte de los Pirineos Centrales meridionales localizada en los limites de la provincia de Huesca. El inventario se realizo siguiendo un transecto de dirección este-oeste a través de la provincia oscense, entre las latitudes $42^{\circ} 19^{\prime}$ y $42^{\circ} 56^{\prime}$ norte. En este trabajo - y a partir de la utilización de procedimientos estadísticos. analizamos las tendencias regionales presentes en la distribución espacial de los glaciares rocosos del área estudiada incidiendo, con particular énfasis, en la importancia que - de cara a un desarrollo diferencial - muestran los aspectos relacionados con la litología y el grado de fracturación.
\end{abstract}

Abstract..- One hundred and seventy rock glaciers were identified in an aerial photograph inventory of $6.500 \mathrm{~km}^{2}$ in the Spanish province of Huesca which includes a major part of the southern Central Pyrenees. The inventory was made on an east-west transect across the province of Huesca between latitudes $42^{\circ} 19^{\prime}$ and $42^{\circ}$ $56^{\prime}$ north. The study was carried out using statistical techniques (quadrat analysis, Poisson distribution analysis and variance-mean ratio test), and demonstrates the existence of evident irregularities that we have related to differences in lithology and fracturing density over the area studied.

Sumario.- I.- Introducción. II.- Distribución espacial de los glaciares rocosos analizados: II.1. Pautas de distribución altitudinal. II. 2. Pautas de distribución espacial (s.s.): II.2.1. Análisis de los cuadrados. II.2.2. Análisis de la distribución de Poisson. II.2.3. Ratio varianza-media. III. Conclusiones. IV. Bibliografía.

\section{INTRODUCCION}

El estudio de la distribución espacial de circos y glaciares rocosos a una escala regional, partiendo de procedimientos estadísticos, ha sido abordado por varios autores (TRENHAILE, A.S., 1975; LUCKMAN, B.H. y CROCKETT, K.J., 1978; ELLIS, J.M. y CALKIN, P.E., 1979; etc.) durante las dos últimas décadas. En la provincia de Huesca, este tipo de trabajo estaba todavía por realizar, aunque ya habían sido llevados a cabo algunos análisis previos (CHUECA, J., 1990, 1991 a). Para su ejecución es necesaria la preliminar identificación de los glaciares rocosos objeto de estudio que, en nuestro caso, fue realizada a partir de fotografías aéreas de un área superior a los $6.500 \mathrm{~km}^{2}$ que incluía la parte de los Pirineos Centrales meridionales localizada en los límites de la 
provincia de Huesca. El inventario se realizó siguiendo un transecto de dirección este-oeste a través de la provincia oscense, entre las latitudes $42^{\circ} 19^{\prime}$ y $42^{\circ} 56^{\prime}$ norte (fig. 1). Para minimizar los problemas de interpretación, partimos de un área para la cual se contaba con fotografías aéreas uniformes y de gran calidad. Fueron tomadas por el I.G.N. durante los primeros años ochenta con una escala aproximada de 1:25.000. Los glaciares rocosos fueron cuidadosamente trasladados a mapas 1: 50.000 (M.T.N. números: 117, 118, 143, 144, 145, 146, 147, 148, 175, 176, 177, 178, $179,180,209,210,211,212$ y 213) desde los cuales se realizaron medidas de la longitud, anchura, área, perfil longitudinal, elevaciones en la zona de raíz y frente, y orientación de cada uno de ellos. En este trabajo, únicamente nos ocuparemos de los aspectos relacionados con la distribución espacial de los glaciares rocosos y las anomalías que en ella hemos detectado ligadas, básicamente, a factores litológicos y al grado de fracturación. Las inferencias paleoambientales derivables del mismo tipo de análisis son objeto de otra publicación (CHUECA, J., 1991 b).

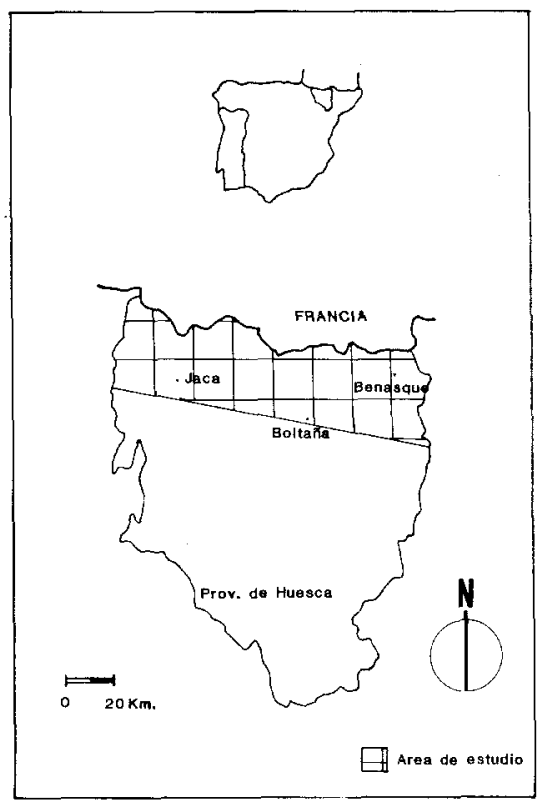

Fig. 1.- Mapa de Situación 


\section{DISTRIBUCION ESPACIAL DE LOS GLACIARES ROCOSOS ANALIZADOS.}

El análisis de la distribución espacial de los glaciares rocosos del área de trabajo fue llevado a cabo siguiendo dos direcciones principales y complementarias. En primer lugar se procedió al estudio de las pautas de distribución altitudinal (a partir del empleo de la técnica del análisis de tendencias superficiales) presentes en los 170 glaciares rocosos catalogados en el ya citado inventario. En segundo, se pretendió confirmar la posible existencia de irregularidades en su distribución espacial (sensu stricto), partiendo de la consideración de los glaciares rocosos como entidades puntuales y de su posterior tratamiento por medio del análisis de cuadrados, análisis de la distribución de Poisson y método del cociente varianza-media.

\section{1. Pautas de distribución altitudinal}

El análisis de tendencias superficiales (trend surface analysis) ha sido aplicado en una gran variedad de campos [DOORNKAMP, J. C. y KING, C.A.M., 1971; CHORLEY, R.J. (ed.), 1972; SHAW, G. y WHEELER, D., 1985], abarcando desde el análisis y reconstrucción de paleosuperficies de erosión (KING, C.A.M., 1969; RODDA, J.C., 1970; DOORNKAMP, J.C., 1972) hasta el estudio de las pautas altitudinales que guían el establecimiento de los circos glaciares y glaciares rocosos (ROBINSON, G., 1972; LUCKMAN, B.H. y CROCKETT, K.J., 1978). Comúnmente se utiliza para determinar la existencia de tendencias espaciales y para identificar - a partir del uso de los valores de residuales - las áreas con un comportamiento local diferenciado o anómalo, en las que la presencia de factores locales anula la tendencia regional. Este tipo de análisis es empleado para crear "superficies" tridimensionales, definibles matemáticamente, a partir de un conjunto de observaciones distribuídas espacialmente de una variable dada (glaciares rocosos, en nuestro caso). En sus propósitos y métodos de ejecución es análogo a los análisis de regresión, y puede definirse como una combinación de métodos no-lineales y múltiples. Sin embargo, mientras que los procedimientos usuales de regresión permiten calcular la variable dependiente en relación al valor numérico de una o más variables predictor $(Y=a+b X)$, en el análisis de tendencias superficiales las estimaciones se hacen teniendo básicamente en cuenta la localización espacial, pudiendo ser definida ésta última a partir de una serie de coordenadas cartesianas obtenidas mediante la cuadriculación del espacio estudiado $(Y=a+b M+c N)$. 
Con nuestro estudio ( $v$. una serie de conclusiones preliminares en CHUECA, J., 1990), pretendíamos confirmar la existencia o no de una tendencia regional en la distribución altitudinal de los glaciares rocosos de esta zona del Pirineo oscense. Para ello, procedimos en primer lugar a obtener - a partir de los datos de altitudes máximas y mínimas en las zonas de raíz y frente, respectivamente - los valores de las altitudes medias de los 170 glaciares rocosos identificados. Posteriormente, superpusimos al área de estudio una cuadrícula (cuyo espaciado interno comentaremos más adelante) y obtuvimos los valores de la tendencia superficial de primer orden a partir del método del valor esperado de Krumbein (altitud media general: 2.320,6 m. s.n.m.). Este método permite realizar una cartografía isoplética de la tendencia regional (fig. 2) que, aun quedando lejos del refinamiento y flexibilidad ofrecido por los procedimientos cuadráticos y cúbicos (posibilidad de un cierto grado de curvatura y un mayor grado de ajuste) es más que suficiente para detectar y plasmar en un mapa la presencia de pautas en la distribución espacial estudiada

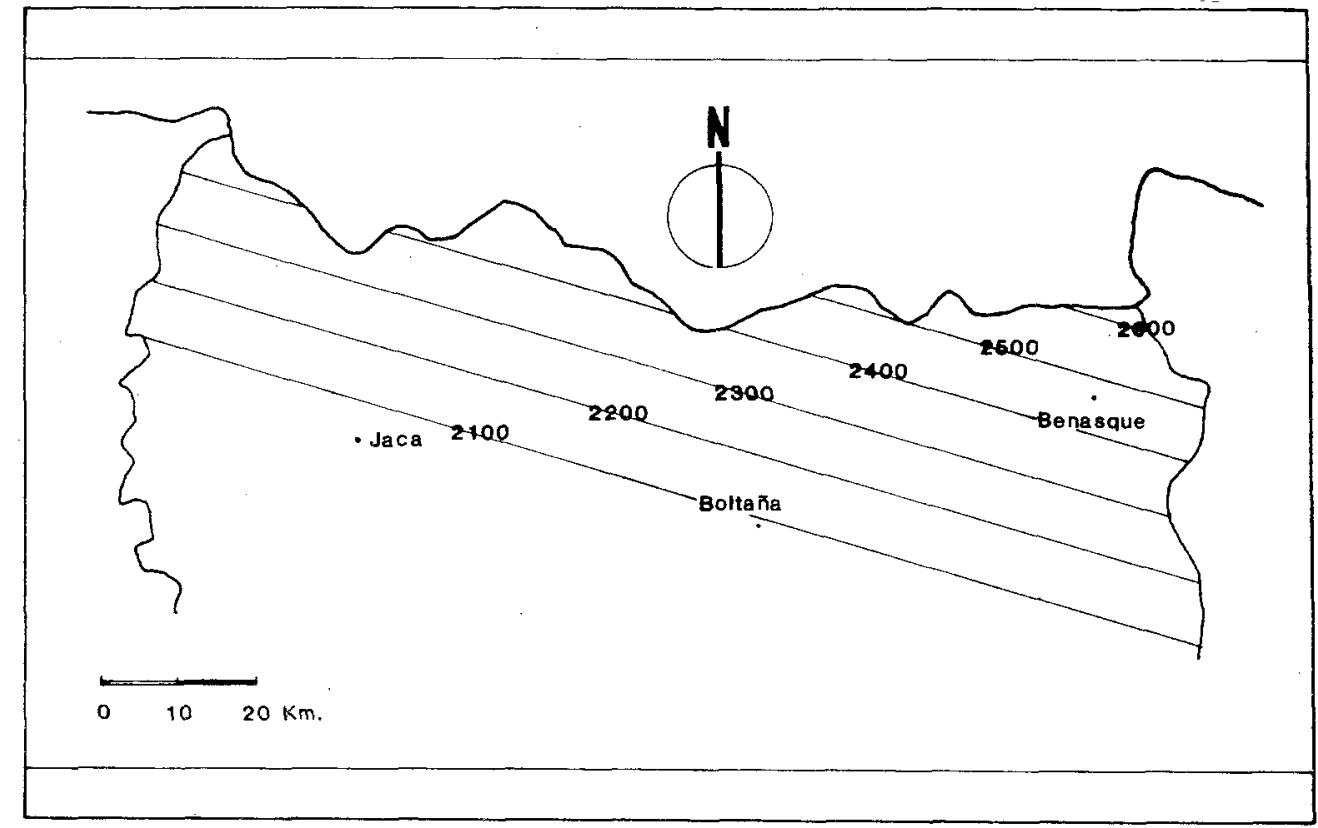

Fig. 2.- Pautas en la distribución altitudinal de los glaciares rocosos en la provincia de Huesca. Mapa de isolíneas obtenido a partir del análisis de tendencias superficiales.

En el área de estudio, además del esperado y lógico gradiente latitudinal, se observó un marcado y general incremento en las alturas medias en dirección E. a lo largo de todo el sector analizado. Podría intentar explicarse este gradiente a partir de un mero control topográfico (presencia - en general - de una tendencia constatable hacia el desarrollo de macizos con mayores alturas 
conforme nos acercamos al interior de la cadena) pero, la localización de un buen número de glaciares rocosos situados fuera del ámbito de los circos nos obliga a desechar per se esa posibilidad. Pensamos, por el contrario, que este tipo de distribución viene motivado por factores estrictamente climáticos (o paleoclimáticos) relacionables con pretéritas condiciones en las que la existencia de unas precipitaciones decrecientes y un marcado incremento de la continentalidad hacia el área oriental se harían evidentes, favoreciendo un desarrollo altitudinal diferenciado en los glaciares rocosos del sector pirenaico analizado. El estudio detallado de la porción oriental del Pirineo meridional, intentando confirmar la existencia de una simetría en la distribución altitudinal de los glaciares rocosos - cuyo eje central de inflexión se situaría, precisamente, en la zona de los Montes Malditos - podría ayudar al apuntalamiento (que queda aquí tan sólo embrionariamente expuesto) de esta hipótesis.

De cara a completar la visión general que nos ofrece el análisis de tendencias superficiales que, no olvidemos, sirve básicamente para generalizar la tendencia regional y filtrar las variaciones locales debidas a las condiciones de localización, orientaciones, tipos de glaciar rocoso, etc.-, fue elaborado el correspondiente mapa de residuales (fig. 3). Los valores de residuales (ESTEBANEZ, J. y BRADSHAW, R. P., 1979) nos indican el grado de ajuste entre los valores reales y los valores esperados, es decir, nos indican la forma en que la función predice la variable dependiente. Estos valores pueden cartografiarse, y observarse de este modo la variación espacial del grado de ajuste del análisis de tendencias superficiales. Así, por ejemplo, la existencia de residuales con valores bajos nos está indicando una estrecha correspondencia entre el valor observado y el estimado. En aquellas zonas en donde la ecuación de regresión estima en exceso, aparecen valores de residuales positivos, y en aquellas otras en las que la ecuación de regresión subestima, se dan residuales negativos.

En nuestro caso concreto, pueden extraerse de su estudio algunas consideraciones particularmente interesantes. Así, los valores concentrados de residuales más bajos (oscilantes entre -4 y -2), indicativos de que los glaciares rocosos establecidos en estas zonas se encuentran a una altitud mucho menor de la que sería lógico esperar partiendo - únicamente - del análisis de la tendencia general, se localizan tanto en la zona más meridional del área trabajada (prolongación oriental de la Sierra de Tendeñera - en torno a la Peña de Otal y al Pico de Fenez - ; Macizo de Cotiella-Sierra de Chía), como en el sector septentrional en las proximidades del Mte. Perdido-Sierra 
de las Sucas. Todos ellos enmarcados, por tanto, dentro de afloramientos calizos de tipo masivo. Por otro lado, existe una cierta tendencia hacia la localización de los valores positivos más altos (+ 2) en las áreas con predominio de rocas graníticas (Macizo de Posets-Punta Millares), en uno de los espacios que presenta mayor densidad de aparatos glaciares de toda la zona analizada. La explicación a estas anomalías puede estar relacionada con dos elementos quizá confluyentes: en primer lugar, con factores de orden lito-estructural, donde el variable grado de resistencia o fisibilidad de la roca fundamental para la creación de las masas de derrubios generadoras de las formas de glaciar rocoso provoque el desarrollo diferenciado en la distribución altitudinal de los glaciares rocosos (sin necesidad, por tanto, de una acción crioclástica excesivamente acentuada, al ser suplida ésta por un grado de fracturación conveniente - y no por ello masivo -). En segundo lugar, la explicación podría venir dada por factores de tipo genético, estableciéndose a priori una conexión lineal entre las zonas con valores de residuales negativos y procesos predominantes de génesis de glaciar rocoso de tipo periglaciar, y aquellas otras con valores positivos, y procesos genéticos estrechamente dependientes de la acción glaciar.

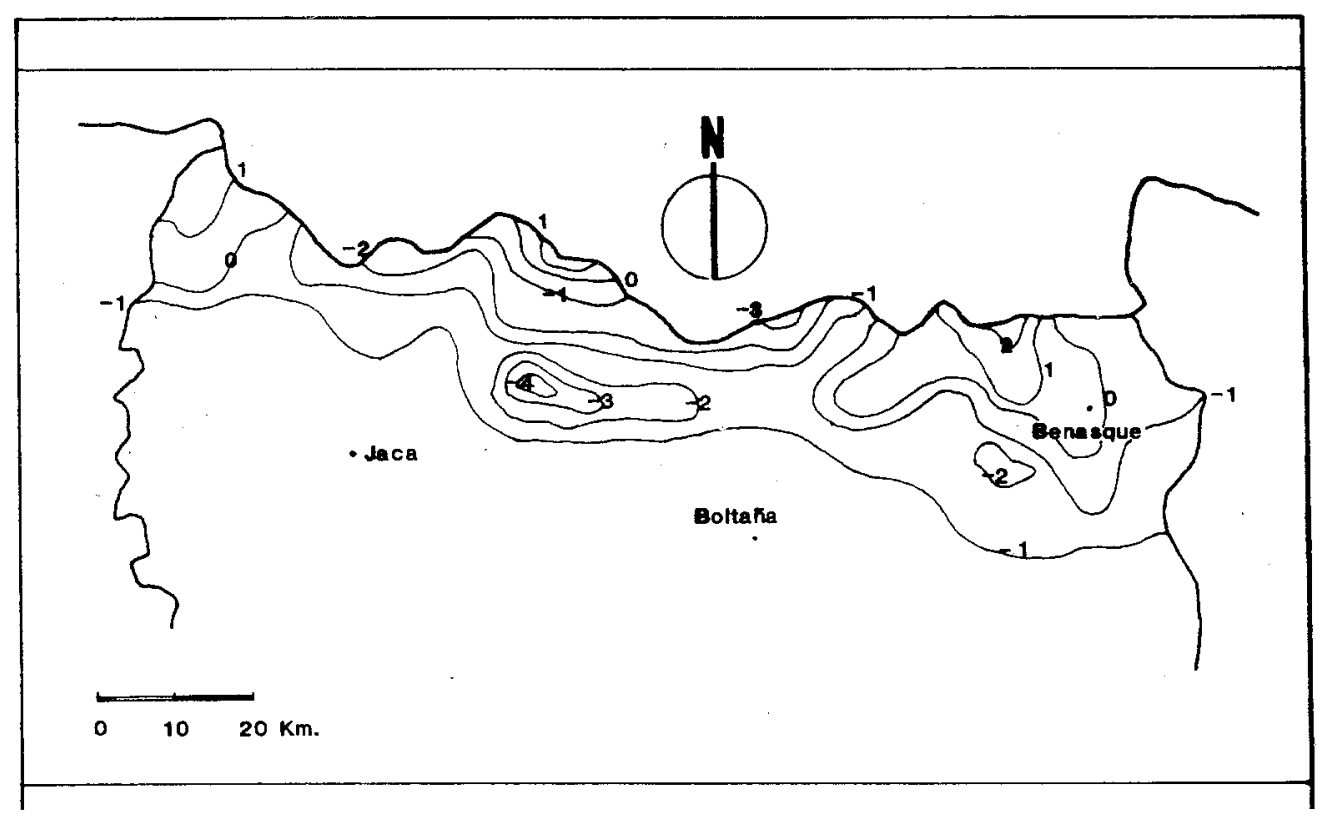

Fig. 3.- Análisis de tendencias superficiales: mapa de residuales. 


\section{2. Pautas de distribución espacial (s.s.)}

El análisis de la distribución espacial de los glaciares rocosos ha sido realizado a partir de su consideración como entidades puntuales, partiendo de la premisa de que cada uno de los puntos (o eventos) debía ser espacialmente discreto y reunir una serie de características básicas necesarias para su ulterior tratamiento estadístico. De cara a confirmar la hipótesis de la existencia de agrupamientos significativos en la localización de los glaciares rocosos del área de estudio llevamos a cabo tres análisis complementarios con los que - por medio de la comparación de distribuciones de probabilidad hipotéticas y distribuciones reales a partir del análisis de los cuadrados, análisis de la distribución de Poisson y ratio varianza-media - intentamos cuantificar y demostrar estadísticamente dicha posibilidad. Para ello se hace necesario, en primer lugar, delimitar cuidadosamente el espacio objeto de análisis y crear la correspondiente red de control. En nuestro caso, elegimos la tradicional malla cuadrangular, al ser una de las unidades básicas de agrupamiento de más fácil construcción y permitirnos la rápida realización de todo tipo de medidas. El tamaño de celdas fue establecido cuidadosamente, al afectar profundamente las variaciones de densidad el carácter de la distribución, a partir del método de la doble área por punto $(2 A / N$, donde $A$ es el área de estudio y $N$ el número de puntos-glaciares rocosos), delimitándose cuadrículas individualizadas de $88 \mathrm{~km}^{2}(9,4 \times 9,4 \mathrm{krn})$.

\section{II.2.1. Análisis de los cuadrados}

- El estudio del análisis de tendencias superficiales nos permitió confirmar la existencia de una serie de irregularidades afectando a la distribución espacial de los glaciares rocosos del área de trabajo. Sin embargo, esas irregularidades podrían haber sido debidas meramente al azar, no estando generadas por ningún tipo de proceso en particular, sino aleatoriamente. Uno de los métodos de análisis espacial que permite diferenciar entre distribuciones de carácter aleatorio y distribuciones reales que pensamos supuestamente que responden a fuerzas y procesos de caracter no aleatorio, es el análisis de los cuadrados.

El método consistió en superponer al área de estudio la ya mencionada retícula de cuadrículas iguales. Una vez superpuesta, se hizo recuento del número de glaciares rocosos presentes en cada una de las celdas, estableciéndose una distribución de frecuencias. La distribución de frecuencias hallada fue coniparada con la distribución de frecuencias teórica de Poisson. Si los glaciares rocosos 
hubieran estado distribuidos uniformemente, habríamos encontrado en cada cuadrícula un mismo número de ellos. Esta hipótesis nula, según la cual no existirían diferencias en el número de glaciares rocosos de cada cuadrícula puede comprobarse aplicando al análisis el test de ji-cuadrado $\left(x^{2}\right)$ :

$$
x^{2}=\sum_{i=l}^{K}\left[\left(O_{i}-E_{i}\right)^{2} / E_{i}\right]
$$

Donde $O$ son las frecuencias observadas y $E$ las frecuencias esperadas. En nuestro caso, el valor crítico de la $x^{2}$ obtenido con 83 grados de libertad y un nivel de confianza de 0,001 fue de 128,55. Al ser este último excedido ampliamente por el valor de ji-cuadrado calculado para el área de estudio $(813,62)$, pudimos concluir que - en esta distribución - los glaciares rocosos se encontraban agrupados, rechazándose por tanto la hipótesis nula.

\section{II.2.2. Análisis de la distribución de Poisson}

El análisis de los cuadrados nos confirma la existencia de una agrupación significativa en la distribución espacial de los glaciares rocosos trabajados. No obstante, y si queremos añadir una información complementaria a la distribución estudiada, se hace necesario calcular otro tipo de tests. La distribución de Poisson se aplica comúnmente a problemas relacionados con eventos de carácter puntual, dándonos la posibilidad de definir su forma matemáticamente. Mediante su empleo puede determinarse el grado de aleatoriedad en la distribución de los glaciares rocosos del área objeto de análisis. Nuestra hipótesis de partida afirmaría que la distribución espacial de los glaciares rocosos de la zona de estudio no es aleatoria, sino que vendría motivada por una serie de causas aún por determinar. Si esta hipótesis fuera correcta, la distribución observada no debería aproximarse a la distribución de Poisson, que tiene carácter aleatorio y en la que los eventos son independientes y se localizan sin ninguna preferencia espacial. Según el test de aleatoriedad apoyado en esta distribución, si $n$ puntos aparecen localizados aleatoriamente dentro de una región constituida por un conjunto de unidades menores y de igual tamaño - en nuestro caso, las 84 cuadrículas que cubren la zona de estudio - la probabilidad de que $x$ puntos se encuentren en cada cuadrícula viene dada por:

$$
p(X)=(\lambda / X !) \cdot e^{-\lambda}
$$


Donde $\lambda$ es el número de glaciares rocosos por cuadrícula; $e$ es la constante $2,7183 \ldots$, y $X$ el número específico de glaciares rocosos. El número de glaciares rocosos por cuadrícula puede variar entre cero y un infinito límite teórico aunque, en la práctica, la distribución se evalúa sólo hasta un límite apreciable (en este caso ocho. Vid. tabla 1). Sin embargo, y dado que únicamente son posibles cantidades íntegras de $X$ (lógicamente, no puede haber fracciones de glaciares rocosos en este contexto), la distribución es discreta, y queda representada en forma de histograma y no de curva. La bajísima probabilidad de localizar ocho glaciares rocosos por cuadrícula, con la perspectiva de probabilidades aún menores para valores sucesivos demuestra porqué en este caso no son necesarios cálculos ulteriores (ya que $\mathrm{p}(0)+\mathrm{p}(1)+\mathrm{p}(2) \ldots \mathrm{p}(\infty)=1$, y la suma de las probabilidades hasta $\mathrm{p}(8)$ es 0,999 , es fácil concluir que la probabilidad de 90 más glaciares rocosos por cuadrícula es tan sólo del 0,001).

\begin{tabular}{|c|c|c|c|}
\hline $\mathbf{X}$ & $\mathbf{X} / \mathbf{X} !$ & $\mathbf{e}^{-\lambda}$ & $\mathbf{p}(\mathbf{X})$ \\
\hline 0 & 1 & 0,1322 & 0,1322 \\
\hline 1 & 2 & 0,1322 & 0,2674 \\
\hline 2 & 2 & 0,1322 & 0,2705 \\
\hline 3 & 1 & 0,1322 & 0,1824 \\
\hline 4 & 0,697 & 0,1322 & 0,0922 \\
\hline 5 & 0,282 & 0,1322 & 0,0373 \\
\hline 6 & 0,095 & 0,1322 & 0,0125 \\
\hline 7 & 0,027 & 0,1322 & 0,0036 \\
\hline 8 & 0,006 & 0,1322 & 0,0009 \\
\hline
\end{tabular}

Tabla 1.- Probabilidades según la distribución de Poisson de localizar glaciares rocosos por cuadrícula.

El siguiente paso es calcular el número de cuadrículas que, en una distribución aleatoria del tipo de la de Poisson, se espera que tengan $X$ glaciares rocosos. Para ello, las probabilidades obtenidas en la tabla 1 para los valores de $0,1,2$, etc. glaciares rocosos por cuadrícula, son fácilmente convertidas en frecuencias esperadas para una distribución de Poisson perfecta de acuerdo con la siguiente fórmula:

$$
E(X)=N \cdot p(X)
$$


Donde $N$ es el número de cuadrículas utilizado en el estudio. Así, una vez obtenidos estos valores, y aplicando el test de Kolmogorov-Smirnov (con el que pueden compararse valores discretos con distribuciones teóricas como la de Poisson) a la confrontación entre el número de glaciares rocosos por cuadrícula observados en la realidad, y el número de glaciares rocosos por cuadrícula que hubiera habido de tratarse de una distribución perfecta de Poisson (aleatoria, por tanto), obtenemos un valor $\mathrm{D}=0,595$. A! exceder esta cifra el valor crítico para el nivel de significación del $0.01(\mathrm{D}=1,63 / \sqrt{ } 84=0,177)$ podemos rechazar la hipótesis nula que afirmaba que las irregularidades observadas en la distribución espacial eran atribuibles al azar.

\section{II.2.3. Ratio varianza-media}

Otra aproximación al problema puede obtenerse a partir del empleo de la ratio varianzamedia de la distribución observada. El valor de la media $(\lambda)$ viene dado por la densidad de puntos observada (2,023 glaciares rocosos por cuadrícula, en nuestro caso) y el de la varianza por la fórmula:

$$
\sigma^{2}=\left(\Sigma X^{2} / \Sigma X\right)-(\Sigma X / N)
$$

Donde $X$ es el número de puntos por cuadrícula y $N$ el número de éstas. Uno de los puntos definitorios de la distribución de Poisson es la equivalencia entre media y varianza. Así, si el cociente varianza-media es uno o tiene un valor cercano a la unidad puede concluirse que la distribución analizada es del tipo Poisson y, en consecuencia, espacialmente aleatoria. El alejamiento de la unidad en la ratio tiende a reflejar tendencias tanto hacia el agrupamiento como hacia la dispersión regular. Los puntos localizados regularmente arrojan valores de varianza muy bajos debido a que la mayoría de celdas contiene un número similar de puntos, con el resultado de ratios varianza-media menores de 1,0. En el otro extremo, distribuciones puntuales con tendencia al agrupamiento proporcionan varianzas muy elevadas al acumularse en un escaso número de celdas buena parte de las entidades puntuales, provocando ratios mayores de 1,0. El grado de desajuste respecto a la unidad puede convertirse en un valor $z$ una vez calculado el error estándar de la diferencia $\left(S E_{X}\right)$ a partir de:

$$
S E_{X}=\sqrt{ }[2 /(N-1)]
$$


Donde $N$ es el número total de cuadrículas, y $z$ equivale al cociente entre la diferencia de la ratio observada y la ratio esperada con el error estándar.

En nuestro caso, el valor de varianza obtenido (tabla 2 ) es $\sigma^{2}=9,682$ y el cociente varianzamedia, 9,682/2,023 = 4,785. Una vez calculado el error estándar de la diferencia $\left(S E_{X}=0,1522\right)$ puede obtenerse el correspondiente valor $z(24,387)$ de cara a examinar el grado de ajuste entre la ratio varianza-media y el valor hipotético unidad ya mencionado. Al nivel de significación del 0,01 (para el cual los valores críticos de $z$ son de $\pm 2,58$ ) las diferencias son lo suficientemente grandes como para concluir que la distribución - al igual que el método del análisis de la distribución de Poisson nos demostró previamente - es concentrada, y el agrupamiento de los glaciares rocosos analizados en distintos sectores significativo estadísticamente, sin obedecer a procesos aleatorios de ningún tipo.

\begin{tabular}{|c|c|c|c|c|c|c|c|c|c|c|c|c|c|c|c|c|c|c|c|c|c|c|}
\hline$(\mathbf{n})$ & 0 & 2 & 3 & 4 & 5 & 6 & & & 9 & 10 & & 12 & 13 & $\overline{14}$ & 15 & 1 & & 17 & 18 & 19 & 20 & \\
\hline (q) & 56 & $\overline{8}$ & $\overline{2}$ & $\overline{5}$ & 2 & 1 & & 0 & 0 & 7 & 0 & 0 & 7 & & $\overline{0}$ & & $\overline{1}$ & $\overline{1}$ & 0 & & 1 & 84 \\
\hline$(\mathbf{X})$ & $\overline{0}$ & \begin{tabular}{|l|l|l}
3 & 16 \\
\end{tabular} & & 20 & 10 & 6 & & 0 & 10 & 10 & 0 & 0 & 13 & $\overline{14}$ & $\overline{0}$ & $\overline{1}$ & & $\overline{17}$ & $\overline{0}$ & 19 & 20 & 170 \\
\hline $\bar{X}$ & $\overline{0}$ & \begin{tabular}{|l|l|}
32 \\
\end{tabular} & 18 & 80 & 50 & 36 & 5 & 0 & 0 & 100 & 0 & $\overline{0}$ & 169 & 196 & $\overline{0}$ & $0 \longdiv { 2 5 }$ & & 89 & $\overline{0}$ & 361 & 400 & 1990 \\
\hline
\end{tabular}

Tabla 2.- Cálculo de la varianza de entidades espaciales puntuales a partir del empleo de cuadrículas ( $\mathrm{n}: \mathrm{n}^{\mathrm{o}}$ de glaciares rocosos en cada cuadrícula; $\mathrm{q}: \mathrm{n}^{\mathrm{O}}$ de cuadrículas con " $\mathrm{n}$ "glaciares rocosos; $\mathrm{X}: \mathrm{n}^{\mathrm{O}} \mathrm{de}$ glaciares rocosos por cuadrícula).

Hasta aquí, el examen de las pautas de distribución espacial de los glaciares rocosos localizados en el Pirineo Central español ha demostrado la existencia de evidentes irregularidades detectadas, tanto a través del análisis de los cuadrados, como del análisis de la distribución de Poisson o de la ratio varianza-media. En nuestra opinión - y haciendo salvedad del factor fundamental al que la aparición de los glaciares rocosos debe su existencia: el clima - uno de los elementos básicos que ha condicionado el desarrollo de los glaciares rocosos en el área de estudio es la presencia de un grado suficiente de fracturación y diaclasado actuante sobre litologías adecuadas.

De hecho, las citadas irregularidades se confirman y reciben una explicación coherente cuando vemos cómo el $55,88 \%$ de los glaciares rocosos identificados se localiza en zonas (fig. 4) con predominio de rocas cristalinas (que constituyen el $6,86 \%$ del total del área trabajada), mientras que 
únicamente el $44,12 \%$ de los glaciares rocosos se instala sobre zonas con otro tipo de materiales $(93,14 \%$ del total del área trabajada). Este proporcionalmente mayor número de glaciares rocosos situados en zonas con predominio de litologías graníticas (Panticosa, Posets, Montes Malditos, etc.) es estadísticamente significativo (nivel de significación del 0,001. Test de ji-cuadrado), y coincidente con los datos aportados por otros autores para los Pirineos (SERRAT, D., 1979; GUTIERREZ, M. y PEÑA, J.L., 1981; CHUECA, J., 1989; MARTI, M. y SERRAT, D., 1990), estando en relación con la presencia - habitual en este tipo de materiales - de una densa red de fracturas y diaclasas que ayuda en la producción de grandes bloques angulosos. El hecho de que buena parte de los afloramientos cristalinos aparezcan en el centro de la cadena, donde ésta alcanza sus máximas alturas y el clima es más extremo podría, sin embargo, introducir una cierta distorsión en estas apreciaciones aunque - como ya expusimos anteriormente - no creemos que llegue a invalidarlas.

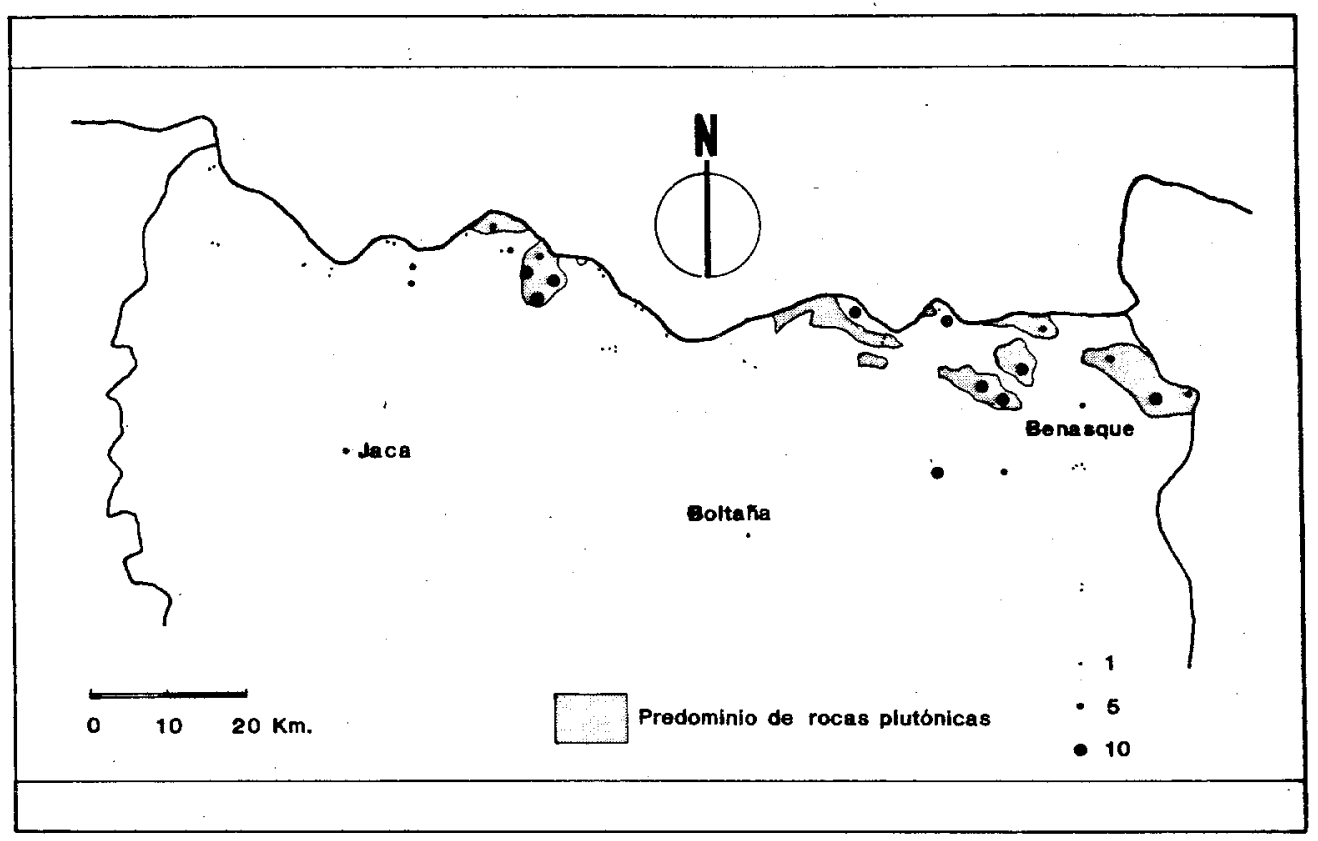

Fig. 4.- Relación entre la distribución de los glaciares rocosos y la litología del área de estudio (los puntos representan glaciares rocosos). 
De cara a evaluar la importancia del factor grado de fracturación, llevamos a cabo un análisis de su desarrollo - perceptible mediante fotografías aéreas - en las zonas localizadas en el área de alimentación de los 170 glaciares rocosos identificados. La densidad de fracturación lineal (expresada en $\mathrm{km} / \mathrm{km}^{2}$ ) varía considerablemente desde valores casi nulos hasta cifras muy altas (fig. 5). Sin embargo, podemos confirmar algunas características generalizables a la totalidad del espacio analizado. Así, el mayor número de glaciares rocosos tiende a concentrarse en los afloramientos rocosos con un alto índice de fracturación (fundamentalmente localizados, como ya hemos citado, en litologías graníticas), situado en torno a $\operatorname{los} 6-10 \mathrm{~km} / \mathrm{km}^{2}$. En la mayoría de los afloramientos calizos (Peña las Once, Chía, Cotiella, Turbón, etc.), el grado de fracturación no es tan elevado y el desarrollo de morfologías de glaciar rocoso decrece claramente. Por otro lado, un grado de fracturación demasiado alto - como el que puede observarse en ciertos sectores con predominio de materiales metamórficos y/o cristalinos - , mayor de $14 \mathrm{~km} / \mathrm{km}^{2}$ parece limitar igualmente la aparición de glaciares rocosos en el área de trabajo.

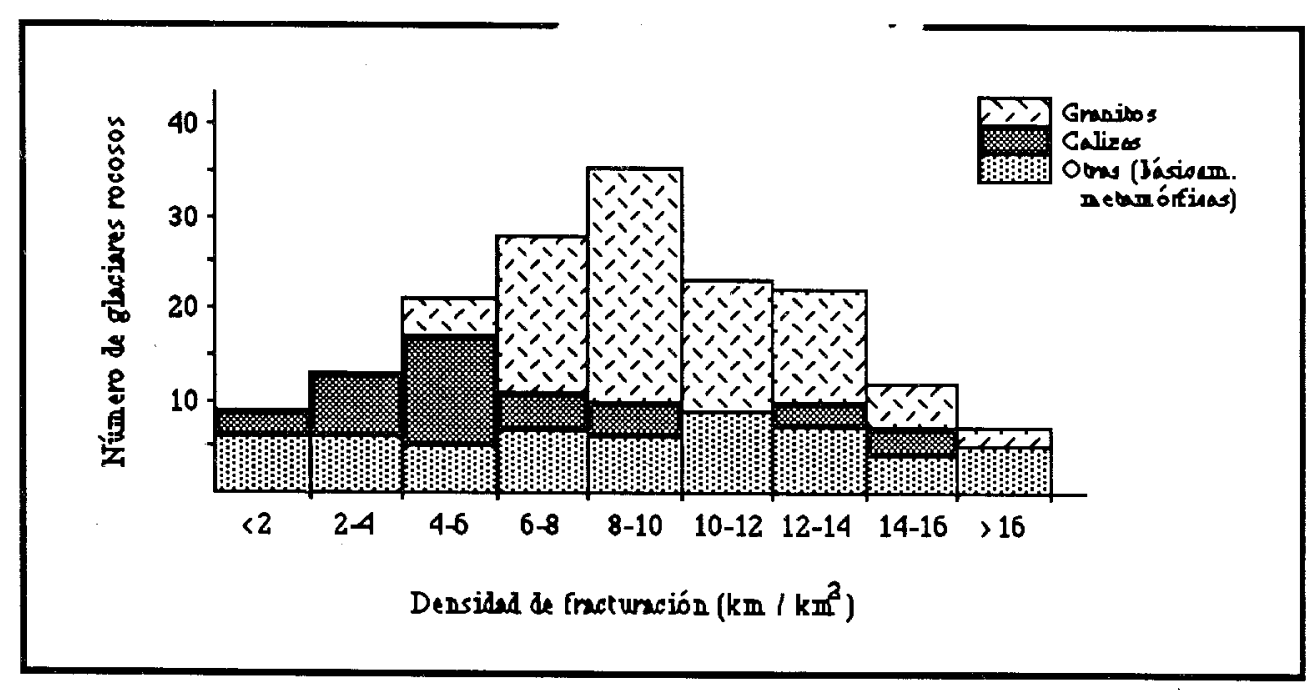

Fig. 5.- Relación entre el número de glaciares rocosos y la densidad de fracturación. 


\section{CONCLUSIONES}

A partir del análisis de las pautas de distribución altitudinal (análisis de tendencias superficiales) llevado a cabo considerando las altitudes medias de los glaciares rocosos localizados en una gran extensión del Pirineo Central, hemos podido definir la existencia de un sesgo en su distribución atribuible a factores de tipo climático-paleoclimático. El estudio del mapa de residuales correspondiente, muestra la presencia de una serie de irregularidades en su distribución espacial que ponemos en relación con factores de orden lito-estructural y genético. En estas irregularidades - tal y como lo confirman el análisis de los cuadrados, análisis de la distribución de Poisson y el método del cociente varianza-media - podemos asegurar estadísticamente que queda totalmente desechada la influencia del azar, siendo el factor litológico (zonas con predominio de rocas cristalinas con un grado adecuado de fracturación) en conjunción, obviamente, con elementos de orden climático

ligados a diferencias altitudinales, los principales causantes de los agrupamientos y concentraciones de glaciares rocosos a lo largo del área de estudio.

\section{BIBLIOGRAFIA}

CHORLEY, R.J., (ed.), (1972), Spatial analysis in Geomorphology, Methuen, London.

CHuECA, J., (1989), Características de los glaciares rocosos del área meridional del batolito de Panticosa (Pirineo oscense), Geographicalia, 26, pp. 61-74.

, (1990), Distribución de los glaciares rocosos en el Pirineo Central (Huesca, España). Una aplicación de la técnica del Análisis de Tendencias Superficiales, Conferencia Internacional sobre el Medio Natural y el Ser Humano en los Pirineos occidentales y Montes Vascos durante el Pleistoceno Superior y Holoceno, Vitoria, pp. 156-157.

(1991 a), Spatial distribution patterns of rock glaciers in the Spanish Central Pyrenees, Permafrost and Periglacial Processes, (en prensa).

(1991 b), Estimación de paleotemperaturas durante el Pleistoceno final: Pirineo Central español, Estudios Geográficos, (en prensa).

DOORNKAMP, J.C., (1972), Trend-surface analysis of planation surfaces with an East African case study; en Chorley, R.J., (ed.), Spatial analysis in geomorphology, Methuen, London.

DOORNKAMP, J.C. y KING, C.A.M., (1971), Numerical analysis in geomorphology: an introduction, Arnold, New York. 
ELLIS, J.M. y CALKIN, P.E., (1979), Nature and distribution of glaciers, neo-glacial moraines and rock glaciers, east-central Brooks Range, Alaska, Arctic and Alpine Research, v.11, pp. 403420 .

ESTEBANEZ, J. y BRADSHAW, R.P., (1979), Técnicas de cuantificación en Geografía, Tebar Flores, Madrid.

GUTIERREZ, M. y PEÑA, J.L., (1981), Los glaciares rocosos y el modelado acompañante en el área de la Bonaigua (Pirineo de Lérida), Bol. Geol. y Min., v. 92, pp. 11-20.

KING, C.A.M., (1969), Trend surface analysis of Central Pennine erosion surfaces, Trans. Inst. Brit. Geogrs., v. 47, pp. 47-59.

LUCKMAN, B.H. y CROCKETT, K.J., (1978), Distribution and characteristics of rock glaciers in the southern part of Jasper National Park, Alberta, Canadian Journal of Earth Sciences, v. 15 (4), pp. $540-550$.

MARTI, M. y SERRAT, D., (1990), Los glaciares rocosos del Pirineo catalán: primeros resultados, I Reunión Nac. de Geomorfología, pp. 191-201.

ROBINSON, G., (1972), Trials on trends through clusters of cirques, Area, v. 4, pp. 104-113.

RODDA, J.C., (1970), A trend surface analysis trial for the planation surfaces of North Cardiganshire, Trans. Inst. Brit. Geogrs., v. 50, pp. 107-114.

SERRAT, D., (1979), Rock glacier morainic deposits in the eastern Pyrenees; en Schluchter, Ch., (ed.), Moraines and Varves, Balkema, pp. 93-100.

SHAW, G. y WHEELER, D., (1985), Statistical techniques in geographical analysis, Wiley \& Sons, New York.

TRENHAILE, A.S., (1975), Cirque elevation in the Canadian Cordillera, Annals of the Association of American Geographers, v. 65, pp. 517-529. 